\title{
Telemonitoring System Communication under Zigbee over a Wi-Fi Network
}

\author{
Smitha K. \\ Department of D.E.C.S, \\ V.T.U, CPGS Bangalore \\ region Muddenahalli, \\ Chikkaballapur, India
}

\author{
SarikaTale, PhD \\ Associate Professor, \\ Department of D.E.C.S, \\ V.T.U, CPGS Bangalore \\ region Muddenahalli, \\ Chikkaballapur, India
}

\begin{abstract}
In this paper a structural analysis in understanding of ZigBee based transmission is investigated and studied. The proposed system is designed and analyzed to achieve a systematic suppressing and load overhead balancing under packet transferring from one mode to another via ZigBee in the absolute presence of $\mathrm{Wi}-\mathrm{Fi}$, the system has successfully achieved a greater rate of success in understanding and analyzing the suppressing ratios of the packets and the same is archived in the results.
\end{abstract}

\section{General Terms}

Telemonitoring, Zigbee, interference, Wi-Fi, patient monitoring

\section{Keywords}

Telemedicine, sensors, packet transfer, Wi-Fi suppressing

\section{INTRODUCTION}

The agenda of the project is to provide a telemedicine approach for rural Indian population, the statistics shows that, in India $78 \%$ of population belong to rural sector among which $48.3 \%$ fail to achieve medical assistance under normal and emergency scenarios. Under the concept of telemedicine and medical remote diagnosis and treatment, a new era of research has been emerged. Data based mining and value based result prediction is possible in today's modern technological era. To achieve these goals, the system is proposed with a clear agenda of providing a connectively gap from rural India to urban and technological society

The demand of telemedicine has recently increased and thus it has seen a random increase under reliability for achieving a trust under this approach. The system is still under processing; hence a motivated spirit has spared to contribute a bit to field. The system is also aimed to achieve high level of patient monitoring. Telemedicine increased a new vision to understand and utilize modern sciences and medical parametics under one roof. This system is proposed with an objective to also contribute to the system under patient monitoring for remote and rural population. Apart from the focused crowd, system is also add-on for urban nuclear families for elderly patient monitoring.

\section{METHODOLOGY}

This section consist of a detailed agenda on system design, existing system and proposed system applications. The chapter is initiated with overall block diagram and then discussing the detailed methodologies involved under implementation.

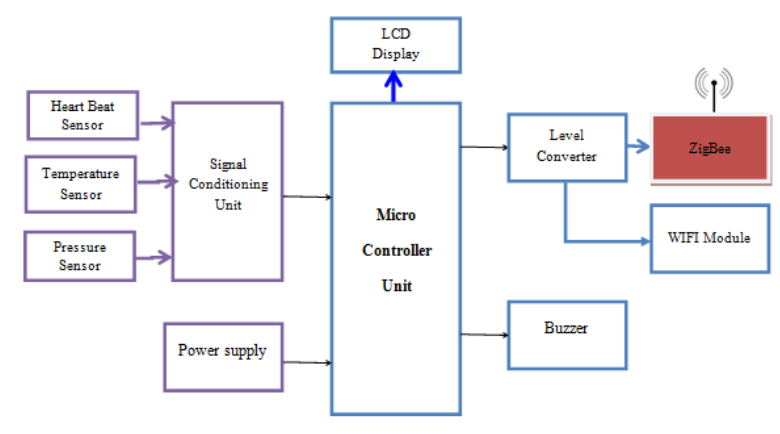

Figure 1: Block Diagram of System Proposed

The overall system design is as seen in figure 1, the system consist a dedicated sensors such as health monitoring. The sensors include the following

\subsection{Temperature sensor}

The temperature sensor is designed and situated for achieving more reliable temperature unit for processing and programming. The temperature achieved is processed and feed into the system model.

\subsection{Pressure Sensor}

The pressure sensor is designed to acquire the sensor reading from the system approach of prediction and sensor. The overall module is to fetch exact value and update the same under the cloud environment for secure and reliable connectivity.

Typically the system is implemented under The Proteus Design Suite is an Electronic Design Automation (EDA) apparatus including schematic catch, recreation and PCB Layout modules. It is produced in Yorkshire, England by Lab center Electronics Ltd with workplaces in North America and a few abroad deals channels. The product keeps running on the Windows working framework and is accessible in English and others

The smaller scale controller reenactment in Proteus works by applying either a hex document or an investigate record to the microcontroller part on the schematic. It is then co-recreated alongside any simple and advanced hardware associated with it. This empowers it's utilized as a part of a wide range of venture prototyping in zones, for example, engine control, temperature control and client interface design. It additionally discovers use in the general specialist group and, since no equipment is required, is advantageous to use as training or educating instrument. Backing is accessible for coreenactment. 
The PCB Layout module is naturally given availability data as a net list from the schematic catch module. It applies this data, together with the client indicated plan rules and different outline computerization devices, to help with blunder free board outline. Plan Rule Checking does exclude fast outline limitations. PCB's of up to 16 copper layers can be delivered with outline size restricted by item arrangement.

\subsection{Algorithmic Modeling}

Algorithm 1: Remote Monitoring System

Input: Sensor data values

Output: ADC converted values

Pseudo code:

1. Initiate the process of collecting and monitoring the environment

2. PIC Controlling Analysis and Controlling Pins model

3. Activation and analysis of sensor values

a. Collect the values of Temperature Sensor

b. Process under the microcontroller unit

c. Pack the values under ADC convertor

d. Pass the control to main

4. Establish the connection to WiFi and then Suppress in the presence of ZigBee and activate the route

5. Pair establishment and processing approach

6. Send the values via ZigBee 2

Algorithm 2: Administrator Monitoring Unit

Input: Signals from the ZigBee sender

Output: TeraTerm

Pseudocode:

1. Collect the incoming request for connection establishment

2. Collect the signals and thus generate the overall path for PC based storage

3. Activate TeraTERM window under Serial to USB port connection and interfacing.

4. Display the values under the scheme

5. Perform suitable action.

\section{RESULTS AND OBSERVATION}

The overall computation delay is computed with the independent observation of packet losses under ZigBee and Wi-Fi ranges. In this section we have considered a Zigbee comparison with the overall Wi-Fi based transferring and thus computing the betterment,

\subsection{Wi-Fi based transferring}

In this segment, the data is continuously sent even in the absence of absolute information. The information is merged and slipped out of focus and thus the Wi-Fi is supposed to be suppressed in the overall computation. The proposed system thus computes this in section 2. In Fig 2 we have demonstrated the overall packet error rate and thus we can summaries it as one of the heavy losses.

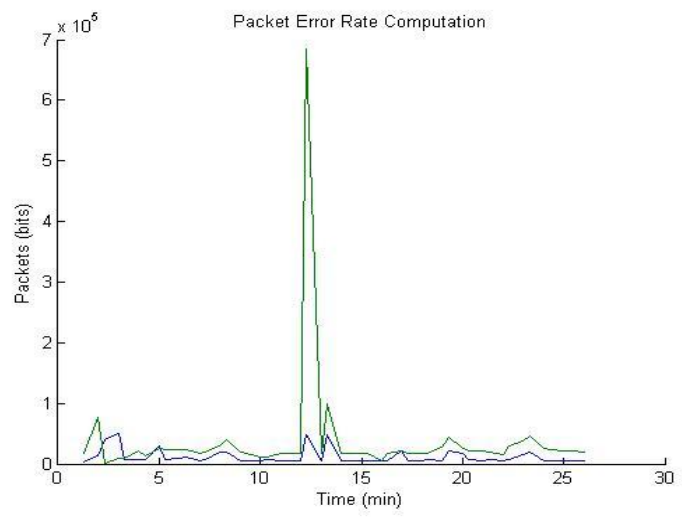

Figure 2: Wi-Fi based packet transfer

\subsection{ZigBee based packet transferring (Proposed System)}

The special mode of transferring is the ZigBee mode, here the channel is compressed and feed into the continues channels and thus the overall transferring is made easy and enhancive for computation and information transformation, the data is highly transferred and this increases the reliability of the system under the proposed method.

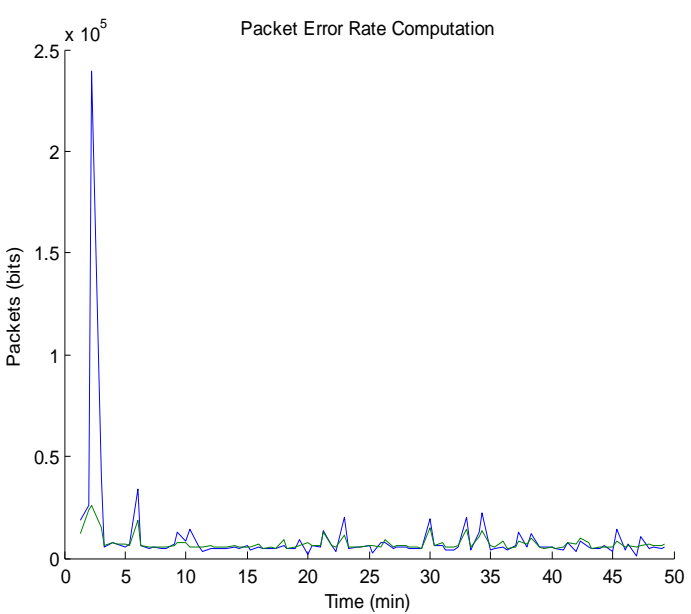

Figure 3: Zigbee based packet transfer

\section{CONCLUSION}

The protective system design under ZigBee based transmission is encouraged and processed in the thesis and proposed system. The overall agenda is to suppress the $\mathrm{WiFi}$ and activate the ZigBee node for transmission and hence it is successfully achieved in the proposed system. To add-on value, the system is upgraded with IP based data uploading to achieve the concept of telemedicine and telemonitoring. This is remote server application management and hence the overall system design is achieved as per the objective.

Apparently, the system is more consistent to the remote monitoring approach of telemedicine as the system consumes low amount of effective data in uploading and storing under the cloud environment, this system is more reliable and has faster accessing rate.

\section{FUTURE ENHANCEMENT}

The system simulated is designed for short range sensors under electronics development unit and automation environment. The system has successfully transmitted the data values to the server o a dedicated IP and thus the simulated graphs are achieved. Hence the system can developed in 
future, the system can be improved with the dedicated portal analysis and port for value collection with respect to independent patient. System reliability can be improved by detailed control sharing by the patients.

\section{ACKNOWLEDGMENTS}

Our thanks to the experts who have contributed towards development of the template

\section{REFERENCES}

[1] IEEE Standard for Local and Metropolitan Area Networks, Part 15.6:Wireless Body Area Networks, IEEE 802.15.6-2012, Feb. 2012.

[2] Wireless Medium Access Control (MAC) and Physical Layer (PHY) Specifications for Low-Rate Wireless Personal Area Networks (WPANs), IEEE 802.15.4a2007, Part 15.4, 2007.

[3] X. Zhang and K. G. Shin, "Cooperative carrier signaling: Harmonizing coexisting WPAN and WLAN devices," IEEE Trans. Netw., vol. 21, no. 2, pp. 426-439, Apr. 2013.

[4] Z. Zhao, G. H. Yang, Q. Liu, V. O. K. Li, and L. Cui, "Implementation and application of a multi-radio wireless sensor networks testbed,"IET Wireless Sensor Syst., vol. 1, no. 4, pp. 191-199, Dec. 2011.

[5] C.Won, J.Youn, H. Ali, H. Sharif, and J. Deogun, "Adaptive radio channel allocation for supporting coexistence of 802.15.4 and 802.11b," in Proc IEEE Veh. Technol. Conf., 2005, pp. 2522-2526.

[6] P.Yi, A. Iwayemi, and C. Zhou, "Developing zigbee deployment guideline under WiFi interference for smart grid applications," IEEE Trans. Smart Grid, vol. 2, no. 1, pp. 110-120, Mar. 2011.

[7] J. Kim and Y. Kwon, "Interference-aware topology control for low rate wireless personal area networks," IEEE Trans. Consumer Electron., vol. 55, no. 1, pp. 97104, Feb. 2009.

[8] R. Francisco, L. Huang, and G. Dolmans, "Coexistence of WBAN and WLAN in medical environments," in Proc. IEEE Veh. Technol. Conf., 2009, pp. 1-5.

[9] J. Misic and V. B. Misic, "Bridging between IEEE 802.15.4 and IEEE 802.11 b networks for multiparameter healthcare sensing," IEEE J. Sel. Areas Commun., vol. 27, no. 4, pp. 435-449, May 2009.
[10] J. Misic and V. B. Misic, "Bridge performance in a multitier wireless network for healthcare monitoring," IEEE Wireless Commun., vol. 17, no. 1, pp. 90-95, Feb. 2010.

[11] S. Rashwand and J.Misic, "Two-tierWBAN/WLAN healthcare networks; priority considerations," in Proc. IEEE Global Telecommun. Conf., 2012, pp. 5398-5403.

[12] M. N. Deylami and E. Jovanov, "A distributed scheme to manage the dynamic coexistence of IEEE 802.15.4-based health-monitoring WBANs,'IEEE J. Biomed. Health Informat., vol. 18, no. 1, pp. 327-334, Jan. 2014.

[13] N. Torabi and V. C. M. Leung, "Realization of public Mhealth service in license-free spectrum," IEEE J. Biomed. Health Informat., vol. 17, no. 1, pp. 19-29, Jan. 2013.

[14] N. Torabi and V. C. M. Leung, "Cross-Layer design for prompt and reliable transmissions over body area networks," IEEE J. Biomed. Health Informat., vol. 18, no. 4, pp. 1303-1316, Jul. 2014

[15] ITU-T recommendation G.1010: End-user multimedia QoS categories. (2001, Nov.). [Online]. Available: http://www.itu-t.org

[16] Z. Yuan and G.-M. Muntean, "A prioritized adaptive scheme for multimedia services over IEEE 802.11 WLANs," IEEE Trans. Netw. Service Manage., vol. 10, no. 4, pp. 340-355, Dec. 2013.

[17] S. Shin, H. Park, S. Choi, and W. Kwon, "Packet error rate analysis of zigbee under WLAN and bluetooth interferences," IEEE Trans. Wireless Commun., vol. 6, no. 8, pp. 2825-2830, Aug. 2007.

[18] Wireshark. (2013, Oct. 28). [Online]. Available: http://www.wireshark.org/

[19] T. Auld, A. W. Moore, and S. F. Gull, "Bayesian neural networks for internet traffic classification," IEEE Trans. Neural Netw., vol. 18, no. 1,pp. 223-239, Jan. 2007.

[20] N. Langhammer and R. Kays, "Performance evaluation of wireless home automation networks in indoor scenarios," IEEE Trans. Smart Grid,vol. 3, no. 4, pp. 2252-2261, Dec. 2012. 